\title{
A Preliminary Study on Connectivism-Constructivism Learning Theory Based on Developmental Cognitive Neuroscience and Spiking Neural Network
}

\author{
Xin Liu, Huailong Li \\ School of Education, Huaibei Normal University, Huaibei, China \\ Email: liuxinhb@126.com
}

How to cite this paper: Liu, X. and Li, H.L. (2021) A Preliminary Study on Connectivism-Constructivism Learning Theory Based on Developmental Cognitive Neuroscience and Spiking Neural Network. Open Journal of Applied Sciences, 11, 874-884. https://doi.org/10.4236/ojapps.2021.118064

Received: July 5, 2021

Accepted: August 16, 2021

Published: August 19, 2021

Copyright $\odot 2021$ by author(s) and Scientific Research Publishing Inc. This work is licensed under the Creative Commons Attribution International License (CC BY 4.0).

http://creativecommons.org/licenses/by/4.0/

(c) (i) Open Access

\begin{abstract}
Based on a new theoretical perspective, this paper attempts to unify the seemingly incompatible learning theories of Connectivism and Constructivism into a scientific theoretical framework. The Connectivism-Constructivism learning theory is not a simple superposition of the two theories. Instead, it absorbs the essence of the learning theory of Constructivism, Connectivism and Neo-Constructivism, and takes the two empirical scientific experimental results of developmental cognitive neuroscience and spiking neural network as the factual basis, and develops two theories from the perspective of development. Integration, to achieve the resolution of contradictions, complement each other, and then rebuild. This paper discusses Connectivism-Constructivism learning theory. The theory holds that the essence of knowledge is the connection between the subject and the environment. There are two forms: physical form and logical form. The only logical form can be realized and utilized by people. Learning can be divided into two stages: connection and construction. Connection is the premise, construction is the core, and the network action generated in the connection stage as a raw material is pruned, and processed by various systems in the construction stage to become psychological representation. When the psychological representation is used, the relevant network shaping is finished, and the meaningful network is formed, which completes the change of knowledge from physical form to logical form and from logical form to physical form. Therefore, learning is the process of constructing meaningful network. We should not only promote the students' connection stage, but also help the students' construction stage. The innovation and breakthrough contribution of this paper is that it is the first time to look at the topic of learning theory from a new re-
\end{abstract}


search perspective. In order to explore a more convincing learning theoretical framework, this article takes the lead in seeking theoretical support and factual basis from developmental cognitive neuroscience and Spiking neural network. As a result, Connectivism learning theory and Constructivism learning theory are successfully integrated into a rather complete and effective theoretical framework to reconstruct Connectivism-Constructivism learning theory.

\section{Keywords}

Connectivism, Constructivism, Learning Theory, Development, Neural Network

\section{Introduction}

With the progress of science and technology and the arrival of the network era, digital learning has increasingly become the mainstream learning method in the network era. Traditional learning theories, such as Behaviorism and Cognitivism, are progressively more difficult to apply to online courses. In early 2005, the Canadian scholar, Simmons in the Connectivism A Learning Theory for the Digital Age, the article puts forward the correlation of Connectivism, it has a profound discipline foundation (e.g., social management, chaology, complexity theory, self-organization theory, brain science, etc.), argues that "all the network-centric, everything is a network of interconnection and interflow", at that time really novel and unique, caused a big stir [1]. Therefore, once the Connectivism learning theory was born, it attracted the attention of researchers at home and abroad, and quickly established its position as the leading learning theory in the network era, used to guide the education and teaching practice in the network era. However, after more than ten years, with the in-depth study of the learning theory of connectionism, education researchers have become more clear about the views, principles and examples of the theory, but they have also begun to reflect on whether the learning theory of connectionism is the most reasonable and perfect answer at present. Nowadays, the arrival of the era of artificial intelligence has further confirmed the correctness of the Connectivism learning theory, which is still the leader of the current learning theory. Connectivism learning theory has brought two key inspirations worth thinking about:

One is that Simmons says "black box", trying to solve his opposition to the previous learning theory view of the human mind, he thinks that the study theory of metaphor exists serious defects of the human mind, Behaviorism, Cognitivism and Constructivism, respectively, compared the human mind to "black box", "computer" and "meaning constructors", and these metaphors are too pale. Simmons writes "... now that we know that the brain is divided into different functional areas, we are gradually opening the black box". However, Connectivism theory, the core elements of "network", and another "computer 
neural network" with high similarity, always hard to be solved in the "black box", so far as to say that "neural network" is the most complex and difficult to interpret the "black box", in the same way, "the brain neural network" in cognitive neuroscience is not before want to so simple and optimistic, a growing body of evidence indicates that although generally exist in functional brain regions, but relatively fuzzy, area clear boundaries between brain areas and there is no one-to-one correspondence relationship function-behavior [2]. Hence, it is impossible to reveal the "black box" simply by relying on the Connectivism learning theory. As we all know, neural network is the representative product of the connectionist artificial intelligence school, and problems such as "black box" and poor interpretability have not been solved, and are still major shortcomings. In this way, even if Simmons, insisted that a computer model is not accurate, neuroscience has revealed that the human mind and computer models are different, but supporters also will inevitably come to the conclusion that those similar to connection school, knowledge is hidden in the network, is in a state of not defined, thus learning as spontaneous training, teaching cannot be preset. Admittedly, this is somewhat the opposite of what Simmons advocates, falling into the "black box to black box" situation. The second is the enlightenment of the "connectivity" described by connectionism in detail. Just as Zhuli Wang mentioned in his elaboration of "Neo-Constructivism", what is connectivity? How to connect? How can we be sure that we are connected [3]? According to Connectivism learning theory, "know who, know where" is connected. If so, people have a lot of contact with teachers, students, online libraries, etc., almost every day. Is learning more effective if we have more of these contacts? Empirically, clearly not. Simmons' definition of "connectivity" is too broad, resulting in a large number of "connectivity", while other indicators of "connectivity", such as quality and efficiency, are easily ignored. Further analysis shows that the root problem actually lies in the "establishment" of connectivity by Connectionism. Simmons and others emphasize "more connectivity and less construction. We often create connectivity", but they ignore the fact that the high-level, uniquely human "connection" is "construction". To take an example of face recognition, face recognition is a social function of the brain, and this ability can be extended between humans and animals, between animals, and even between non-living things (such as computers) [2]. Thus, the relational learning theory has not yet faced up to the inseparable relationship between "establishment" and "construction". Because of the lack of psychological construction, Connectivism learning theory still has a lot of problems that cannot be solved, but it is still indispensable nourishment for subsequent learning theory. In particular, it is often accessible, rather than illusory, and contains multidisciplinary results, which should serve as a solid theoretical foundation.

This topic is the study of learning theory. The basic theory of learning has always been the top priority in the fields of cognitive psychology, learning psychology, educational technology and so on. It has a very important value and requires persistent efforts. This study preliminarily discusses the Connectivism- 
Constructivism learning theory. Its theoretical significance lies in that, on the basis of previous studies and based on a new theoretical perspective, it integrates the Connectivism learning theory and the Constructivism learning theory into a scientific theoretical framework to supplement and improve the relevant theoretical achievements. In reality, Connectivism-Constructivism learning theory can better serve education and teaching practice and produce more practical application products.

The motivation of this paper stems from two points. One is that after years of development and verification, the differences between Connectivism learning theory and Constructivism learning theory are growing. The supporters of the two sides hold different opinions. The academic community urgently needs a new learning theoretical framework that can accommodate the two. Second, Developmental cognitive neuroscience and Spiking neural network, the two subjects gradually rise, and the related research presents an explosive trend. After a large number of literature review, it is found that the two subjects are of great significance to the study of learning theory, which has not been recognized in previous research. For a long time, the research on learning theory is too narrow due to the lack of timely change of research perspective, and often lacks more convincing and quantitative research methods and means. This paper uses the concept and technology of Developmental cognitive neuroscience and Spiking neural network to overcome these problems in previous research and improve the related work.

\section{The Knowledge View, Learning View and Teaching View of Connectivism-Constructivism Learning Theory}

Learning theory often discusses its knowledge view, learning view and teaching view, and Connectivism-Constructivism learning theory is no exception". Meaningful Network" is the central concept of this theory. Based on Ausubels theory of "Meaningful Learning", it is defined as the substantial connection between learners' existing cognitive structure and new knowledge, and many connections and combinations (network) are generated.

The construction of meaningful network refers to the process of establishing substantive connections between learners' existing cognitive structures and new knowledge, and generating many connections and their combinations (networks). In short, learning is the process by which individuals generate meaningful networks. In learning activities, those common contact connections that can be seen everywhere, rough, spontaneous, and never received too much attention cannot be called meaningful networks, but only those connections and combinations that have obtained mental representations and are actively generated by individuals are.

By electroencephalography (EEG) experiments of cognitive neuroscience and the simulation experiments using neural computing model, the existing evidence to show the meaningful network: the brain neural network after accept certain operations (such as pruning), create a very different from before, has the signifi- 
cance of network, the network of neurons cluster synaptic stability, relevant instances of connection, the relevant instances of connection is abate, specificity, further learning effect is obviously promoted.

The knowledge view, learning view and teaching view of Connectivism-Constructivism learning theory are as follows:

1) Knowledge view: the essence of knowledge is the connection established by the subject in the interaction between the subject and the object, which is stored in the neural network of the brain. The brain's neural network is like a gated circuit that can be programmed to change its own pathways, and its physical and logical structures exist side by side. Knowledge also has two forms. The first form, also known as the "original state", is liked to the electrical signal in the physical circuit, which is the concrete physical form of knowledge. It is affected by many factors such as the basic structure of the network and the strength of the connection between nodes, and is characterized by unpredictable, difficult to control and difficult to explain. By the characteristics of knowledge in the form of the first known, however, they are difficult to directly extract, people use, must go through certain system (e.g., language system, memory system) for mental information processing, namely to cutting, splicing of network components, make the connection mode, the intensity is stable, so, connection to become more organized, order, and then complete the transition of the meaningful connection. The abstract logical form of knowledge emerges, which is composed of the first form and the second form ("steady state"). It is constant and reliable, easy to adjust and explicable. The knowledge of the second form is just like the characteristic equations contained in the circuit path. After experiencing the active processing of various systems, the electrical signals are transformed into mental representations, that is, the cognitive structures based on symbols. After the steady-state knowledge is used, it will return to its native state and flow back to the network again, and the meaningful network will be successfully constructed.

2) Learning view: learning is the process of generating meaningful networks by individuals, which can be divided into three stages: connected, constructed and re-connected, and the corresponding knowledge forms of the stages are "original state"-"steady state"-"original state". Connectivity is the forming stage of nodes and connections in a network. Construction is the phase of pruning the network to make sense; reconnection is the stage at which meaningful networks are finally obtained. Individual and situation are the two most fundamental elements of learning, and all learning should be viewed from the perspective of development. The connections generated in the connected stage have a direct impact on the construction of the second stage and are the original materials for construction. Not all the connections are suitable for the next construction, and improper connections will affect the construction stage instead. Then, a large number of connections do not mean a good learning effect. If no meaningful connections are shaped, the learning effect will be poor, or even the learning may not be achieved. Similarly, if the strength of connection is only strength- 
ened extensively, instead of the strong and weak parts, the learning effect will not be good. Learn to carry out more meaningful connection-construction.

3) Teaching view: teachers should help students to generate meaningful networks and assist students to generate more meaningful connections. We should pay attention to the connection stage of learning and the construction stage of learning, cannot care about one or the other, we must first take care the strength of the students in the study of the formation of the connection, length and so on is appropriate, and then adopt the corresponding strategy to promote the construction of these connections.

Indeed, many elements of the Relevance-Constructivism Learning Theory have not been mentioned, and only a general description of the basic parts of the theoretical framework. For example, the teaching strategy, learning strategy, classroom mode and other contents of the theory are the work to be done in the future.

\section{Theoretical Support and Factual Evidence of Connectivism-Constructivism Learning Theory}

\subsection{Theoretical Support}

The theoretical support of Connectivism-Constructivism learning theory mainly includes Connectivism, Individual Constructivism and Neo-Constructivism. The previous article briefly analyzed some questions about the learning theory of connectionism. Here, the three learning theories are briefly summarized, and the key points are selected for analysis and comparison.

Connectivism learning theory introduces the concept of knowledge flow, regards knowledge as the oil flowing in the pipeline, going back and forth between networks. The theory insists that in the age of knowledge explosion, the pipe is more important than the content in the pipe. Knowing where is more important than knowing what is [4]. Such a view has to be said to be puzzling, and it is suspected of putting the cart before the horse. First, the so-called channel of the Connectivism learning theory is also the carrier of information transmission. The topic of "which is more important than the content or the form of information" has long been discussed by communication and media studies, and it is rarely concluded that "the form of information is more important than the content”. Second, research from cognitive neuroscience doesn't quite fit this idea either, showing that there is no priori representation in most of the cortex, and that the plasticity of the cortex is largely dependent on input data-that is, the input data becomes more important than the original network pathways of the cortex [2]; thirdly, some scholars commented after excavating the thoughts of connectionism, "the core idea of the learning theory of connectionism is that knowledge exists in entities outside the learner himself (such as computers) [5]", clearly, this is an Connectivism learning theory inference, but enough for puzzling, both scholars of pedagogy and psychology think, learning is a process of individual development, according to correlation judgment, the learning theory 
of knowledge in the outside world, the individual learning, like apples, get knowledge, and then digest, obviously too objectivism, no human, "the knowledge" can only save? The most important concept categories of Connectivism learning theory are summarized as network, node and connection. Its learning view is just like what Simons said, "Learning is the process of forming a network, and our mind is a network and an ecology. [6]" Thus it can be seen that the Connectivism learning theory overemphasizes the external process while neglects the internal process of the individual. In the final analysis, it fails to grasp the meaning of "development".

On the other hand, Constructivism learning theory corrects the misconceptions left by Connectivism learning theory. Constructivism learning theory clarifies the true meaning of "development", "development is the process of the interaction between the individual and the environment". Constructivism learning theory also has different voices from different angles. According to Piaget's personal constructivism, learners' cognitive development is driven by their relatively independent thinking and operation. According to Vygotsky's social constructivism, the motivation of learners' cognitive development comes from cultural and social interaction and depends on the social basis of thinking. Jonathan is a representative of radical constructivism. He argues that knowledge is not imported from outside, but in people's minds. The subject plays a decisive role in the construction of knowledge, which shows a strong subjectivism color. In fact, in combination with the conclusions of cognitive neuroscience in recent decades, although Piaget's constructivism is the most "ancient", it is the most relevant and appropriate "developmental" learning theory, while the rest of the learning theories are somewhat biased. The reason why social constructivism cannot be accepted by people can mainly refer to the development process of human functional brain. The essence of social basis of thinking is "social brain". The research on the neural basis of infants finds that the reason of social brain is the interaction between relatively primitive brain system, cortical area and environment. Moreover, the "social brain" for face recognition, for example, is not acquired through social use of the skills, but because babies have an innate preference for faces, which means that social interactions are unlikely to be a source of individual cognitive development [2]. Jonathan's radical constructivism is a powerful rebuttal to the learning theory of connectivism, but it should be treated conservatively because its assumptions are closer to the subjectivism of cognitive theory. Actually, Connectivism learning theory absorbs the nutrition of the most social constructivism, as a result, Simmons presents a do not seem to match point, "learning network will eventually be internalized as internal knowledge network exists in our mind, reach the balance of self-organization and his organization", the academics have pointed out that when the limitation in knowledge network, the extent to which Piaget for similar dynamic equilibrium process of constructivism in the scheme, only the starting point and different emphasis, constructivism study is focused on, in the face of new situation, how to through assimilation and adaptation mechanisms, such as making final sche- 
ma formed [6]. It is difficult for Simmons to answer two questions clearly within his own theoretical framework: 1) What is the fundamental difference between the "internalization" of connectivity and the "internalization" of construction? 2) The final form of knowledge is ecological network. Then, when people use knowledge to solve problems of causal logic, how do cognitive structures such as comparison and explanation present themselves? According to Piaget's Constructivism learning theory, thinking is the internalization of action, the causal relationship of cognition comes from the whole of perception-movement, and there are special cognitive tools to manipulate mental images to form cognitive structures.

Connectivism-Constructivism learning theory has adopted many thoughts of Zhuli Wang's Neo-Constructivism, especially the criticism of the Connectivism learning theory. When talking about the relationship between connectivity and construction, there are two important inspirations to the Connectivism-Constructivism learning theory: 1) over-connectivity can lead to information overload, which shows that the amount of connectivity is not the same as the utility of connectivity; 2) there is no better connection without construction, which illustrates the importance of construction for connection [3]. However, NeoConstructivism, still fails to combine the learning theory of connectivism and constructivism. Relevance-constructivism learning theory clearly points out that for "building" a network, "construction is a high-level and unique construction for human beings, and construction is always the core concept of learning". Some scholars have held similar views when studying the application of the theory of connectivism to individual learning environments. They have formally stated that "individual learning environments should attach importance to the construction of learning networks and form meaningful connections" [7]. As for the metaphor of learning, the most appropriate one is Bartlett' $s$ schema assimilation, which was adopted by Mayer in his book Applied Learning Science. Learning is a construction process of schema assimilation, just as learners reconstruct stories on the basis of previous general organizational schema and some story fragments after acquiring new information [8].

\subsection{Factual Evidence}

Undeniably, Piaget's Constructivism learning theory still has some shortcomings. Although his theory should be regarded as the most appropriate theory of cognitive development, it has not had very effective research methods and tools, leading to scholars' criticism that it lacks sufficient convincing evidence (small-scale behavioral experiments are really unsatisfactory). Connectivism-Constructivism learning theory mainly looks for actual experimental results as evidence from developmental cognitive neuroscience and spiking neural network, which are also interdisciplinary integrative subjects. Developmental Cognitive Neuroscience mainly provides experimental evidence on the neurobiological and neuroanatomical, development of functional brain in newborns and other animals before and after birth, supporting the Connectivism-Constructivism learning theory and 
its derivatives. Spiking neural network mainly provides cutting-edge achievements in neural computing, neurodynamics, and brain-like computing, and is used for the construction and verification of models related to cognitive learning to enhance the credibility, feasibility and practical value of the research. The following is a brief overview of the basic points of selection.

Developmental cognitive neuroscience, is also the Constructivism cognitive neuroscience is an understatement, has two meanings [2]: 1) the study of cognitive neuroscience from a developmental perspective. It follows Piaget's constructivism and believes that biological structure is the self-organizing product of the complex interaction between genes and environment. Only by considering the gradual construction process of information can we understand the relationship between the initial state and the final product. This construction is a dynamic process with multiple factors acting together; 2) adopt the cognitive neuroscience approach to research and development. As mentioned above, Piaget's constructivism can only be more convincing with the help of brain science, whose evidence is also an opportunity for the learning theory of related-constructivism. Existing event-related potentials (ERP), functional magnetic resonance imaging (FMRI), and electroencephalography (EEG) technologies are all excellent research means. Here are two proofs of Piaget's constructivism. Milner et al. suggested that cells in the parietal cortex were neither sensory nor motor, but sensorimotor. They are involved in translating sensory information from the retina into motion coordinates and perceptual output into action behavior [2]. Baby's EEG studies have shown that there is a kind of intense timing by high frequency electromagnetic oscillation burst of gamma frequency (about $40 \mathrm{~Hz}$ ) EEG pulse, reflects the object of visual processing "perception", there is an obvious of 8 months old babies this gamma waves, and the other a set of smaller baby widespread higher trend this gamma waves, does not constitute a complete excitation, which further confirmed that the "object permanence" argument [2]. While discussing the functional sex of the human brain development, the development of cognitive neuroscience is more with the "Interaction Specificity" point of view, this kind of orientation is more income than "Mature Theory" or "Skill Learning Theory", that is to say, some cortex may function is not sure at the start, but after a number of different background and tasks, these areas are partially activated, and in the process of development, the brain neural network after selectively clip, depends on the activities of the interaction between brain areas will make the functional differentiation in various regions and this makes these areas more specific, more and more suitable for some kind of narrow situation, in the end can only be limited to the specific situation, is called "regionalization". The idea of "Interaction Specificity" is often called neuroconstructivism, and it is also a cornerstone of Connectivism-Constructivism learning theory.

Spiking (Pulsed) neural networks, known as the third generation of artificial neural networks, are very different from previous artificial neural networks, aiming to learn like the human brain, such as neurons firing pulses of electrical waves. At once, although spiking neural network is not used much, but the re- 
search is becoming increasingly popular. In the long term, spiking neural network must be one of the most promising artificial intelligence technologies, and its importance and value will surpass the traditional artificial neural network. Spiking neural networks are used to make computational models based on Connectivism-Constructivism learning theory, and can also be used as corresponding theoretical arguments, as illustrated here.

Mne-python is an open source third-party Python extension library, which is specially used to process and analyze neurophysiological data. Some researchers have used mne-python in combination with spiking neural network. For example, the specific steps can be as follows: firstly, the BSA spiking sequence coding algorithm is applied to the input coding processing of EEG signals data, then the encoded EEG signals data is sent to the builded spiking neural network model for classification and recognition, and ultimately, the mne-python is used to visually analyze the output results of EEG signals. This work strongly proves that from the perspective of computer engineering, it is appropriate and efficient to the idea of using spiking neural network to justify learning theory.

A classic spiking neural model-Hodgkin Huxley model $(\mathrm{HH})$ model, this model can be regarded as the expansion of the circuit, even if the circuit has no fixed mathematical formula, but already cover the leakage passage, sodium ion channels $(\mathrm{Na})$, potassium $(\mathrm{K})$ differential equation of ion channels such as meaning, that is, the circuit has the characteristics of the pulse neurons [9]. HH neuron model, more precisely, for a given membrane potential ( $V$ ), each channel has a corresponding opening and closing rate. The opening or closing of these channels is described according to the function established in the experiment. These sum functions can be used in differential equations, $\alpha_{n}$ and $\beta_{n}$ describes how the proportion of open channels changes over time $(t)$ :

$$
\frac{\mathrm{d} n}{\mathrm{~d} t}=\alpha_{n}(V)(1-n)-\beta_{n}(V) n
$$

In practical applications, because $\mathrm{HH}$ neuron model needs to use differential equations to represent the model, the calculation is more complex, and it is difficult to carry out large-scale simulation. LIF (Leaky Intergrate and Fired Model) neuron model is often used to replace it in experiments. However, it accurately describes the biological characteristics of membrane voltage, which can be well consistent with the electrophysiological experimental results of biological neurons.

Another study more meaningful, the brain needs sleep to improve work efficiency and memory, but the previous artificial neural network system can't reflect the role of "sleep", and one recent findings such as Watkins, they develop the pulse of neural network system, continuous, after a period of unsupervised learning becomes unstable, if put it in is similar to the human brain after experienced fluctuations in sleep state, will soon restore stability [10]. Spiking neural network can efficiently complete the quantitative research or simulation experiments involving Connectivism-Constructivism learning theory, and then prove 
this learning theory. So, the theory can be applied.

\section{Conclusion}

To sum up, this paper initially obtains a new learning theoretical framework, Connectivism-Constructivism learning theoretical framework, including knowledge view, learning view and teaching view. The core point of view is "Learning is the process of building meaningful network". This paper uses a lot of words to explain the meaning of this point from multiple levels, and gives a similar experimental scheme to verify the new theory, using MNE Python + spiking neural network to analyze EEG signals. The inspiration of this paper comes from the integration of Developmental cognitive neuroscience and Spiking neural network, which is also the theoretical support and factual basis of ConnectivismConstructivism learning theory, The spiking neural network provides the computational framework, mathematical model and neural learning algorithm which are needed to test the learning theory and conform to the biological reality.

\section{Conflicts of Interest}

The authors declare no conflicts of interest regarding the publication of this paper.

\section{References}

[1] Siemens, G. (2005) Connectivism: A Learning Theory for the Digital Age. Instructional Technology \& Distance Learning, 2, 3-10.

[2] Johnson, M. (2007) Developmental Cognitive Neuroscience. Beijing Normal University Press, Beijing.

[3] Wang, Z.L. (2011) Connectivism and Neo-Constructivism: From Connectivity to Innovation. Journal of Distance Education, 29, 34-40.

[4] Siemens, G. (2005) Knowing Knowledge. Philosophy \& Phenomenological Research, 5, 341-344. https://doi.org/10.2307/2102859

[5] Zhang, X.M. (2012) Review on Relevance Theory. Open Education Research, 18, 44-49.

[6] Liu, J. (2014) Research on Relevance Theory of Network Learning and Development of CMOOC Practice. China Educational Technology, 42-48.

[7] Zheng, S.H. (2014) Ecological Concept, Elements and Structure of Individual Network Learning. Journal of Distance Education, 32, 99-105.

[8] Mayer, R.E. (2016) Applied Learning Science. China Light Industry Press, Beijing.

[9] Lindblad, T. and Kinsey, J. (2017) Image Processing and Pulse Coupled Neural Network-Implementation Based on Python. (3rd Edition), National Defense Industry Press, Beijing.

[10] Watkins, Y., Kim, E., Sornborger, A., Kenyon, G.T., et al. (2020) Using Sinusoidally-Modulated Noise as a Surrogate for Slow-Wave Sleep to Accomplish Stable Unsupervised Dictionary Learning in a Spike-Based Sparse Coding Model. 2020 IEEE/CVF Conference on Computer Vision and Pattern Recognition Workshops (CVPRW), Seattle, 14-19 June 2020, 1482-1487.

https://doi.org/10.1109/CVPRW50498.2020.00188 